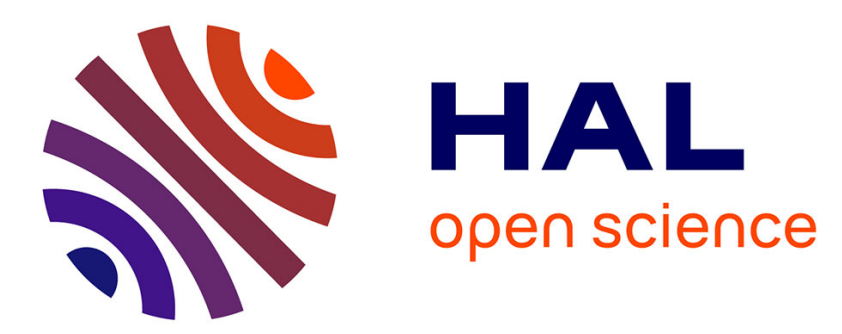

\title{
Feedforward Inertial Actuation for Roll Stabilization of an Underactuated Underwater Vehicle
}

\author{
Divine Maalouf, Vincent Creuze, Ahmed Chemori, Olivier Tempier
}

\section{To cite this version:}

Divine Maalouf, Vincent Creuze, Ahmed Chemori, Olivier Tempier. Feedforward Inertial Actuation for Roll Stabilization of an Underactuated Underwater Vehicle. International Journal of Robotics and Automation, 2015, 30 (1), pp.4161-4196. 10.2316/Journal.206.2015.1.206-4161 . lirmm-01119880

\section{HAL Id: lirmm-01119880 \\ https://hal-lirmm.ccsd.cnrs.fr/lirmm-01119880}

Submitted on 6 Jun 2019

HAL is a multi-disciplinary open access archive for the deposit and dissemination of scientific research documents, whether they are published or not. The documents may come from teaching and research institutions in France or abroad, or from public or private research centers.
L'archive ouverte pluridisciplinaire HAL, est destinée au dépôt et à la diffusion de documents scientifiques de niveau recherche, publiés ou non, émanant des établissements d'enseignement et de recherche français ou étrangers, des laboratoires publics ou privés. 


\title{
FEEDFORWARD INERTIAL ACTUATION FOR ROLL STABILIZATION OF AN UNDERACTUATED UNDERWATER VEHICLE
}

\begin{abstract}
Divine Maalouf*, Vincent Creuze*, Ahmed Chemori*, and Olivier Tempier*
\end{abstract}

\begin{abstract}
This paper deals with a novel control architecture for roll stabilization of an underactuated underwater vehicle. This method relies on the use of an internal rotor to generate inertial counter torque in order to cancel out the disturbances affecting the roll. Moreover, the dynamic model incorporates the thrusters' dynamics, and undesirable effects are compensated by a feedforward approach. The performance and efficiency of the proposed control scheme are illustrated by simulation results.
\end{abstract}

\section{Key Words}

Underwater vehicles, Underwater robotics, Mechanical Systems, Inertial Stability

\section{Introduction}

Underwater vehicles have recently attracted a great deal of interest from scientists, engineers, industries and control theorists. These various communities envision in this technology a very

${ }^{*}$ LIRMM, University Montpellier 2 / CNRS, 161 rue Ada, 34095 Montpellier, France; email: vincent.creuze@lirmm.fr , ahmed.chemori@lirmm.fr, olivier.tempier@lirmm.fr - Manuscript recieved 15 July 2013 
useful tool for undersea exploration and complex missions. Depending on the mission needed, various types of vehicles can be used. However, their size has a great impact on their stability. In the case of big underwater vehicles (weighing more than $100 \mathrm{~kg}$ ), the inertia combined with a poor power/mass ratio contributes to increase the vehicle's stability. Inversely, the inertia of small underwater vehicles (weighing less than $30 \mathrm{~kg}$ ) renders them more sensitive to external disturbances (shock, hydrodynamic effects, etc...). Moreover, such a class of vehicles often offers a high power/mass ratio, thus increasing manoeuvrability but also leading to internal disturbances due to the dynamical effects of the thrusters themselves. In fact, the inertial counter torques, as well as the gyroscopic effects produced by the motors and the propellers, induce variations in the robot's attitude. These variations are caused by the disturbing effects coming from the acceleration of the motors. In addition to these effects, the propeller torque can be seen to have the most important impact on the vehicle's orientation. Indeed, most of the above listed effects have been already incorporated in various applications of aerial vehicles [1], but rarely been considered in underwater vehicles. The main reason behind this omission is the presence of nonlinear hydrodynamic and viscous effects considered predominant and the usual use of big sized vehicles attenuating the disturbances caused by the thrusters. Nevertheless, some control methods based on gyroscopic stability and internal actuation have been used in underwater systems. They were firstly investigated in $[2][3][4][5]$ where the behavior of the open-loop was analyzed. Physical motivation was used to exploit geometry in order to stabilize unstable motions. From this study, the idea to use internal actuation to stabilize an underwater vehicle was born. A reduced model of an ellipsoidal vehicle having one or two rotors inside was initially proposed [6]. Then, a full model was presented and validated through simulation results [7][8][9]. This ongoing research of internal momentum exchange led to the development of the underwater vehicle IAUMBUS [10]. A scheme for attitude control based on gyroscopic torques was presented in [11] and [12]. Four control moment gyros units arranged in a pyramid configuration were introduced inside IKURA, a zero-G vehicle (its center of buoyancy and gravity are coincident). It was the first robot able to dive with a vertical 
pitch and then surface in surge. Our interest is directed towards less conventional control methods of small underactuated vehicles based on a more complete model of their dynamics. The control method of interest falls among the less conventional schemes that would use the nonlinearities of the model emerging from the inertial counter torques as a mean of stabilization rather than neglecting them. The study performed in this paper uses a small underwater vehicle unactuated in roll. The proposed solution aims at stabilizing the roll, while compensating for the undesirable effects caused by the thrusters. The method relies on the use of an internal inertial disk, whose acceleration stabilizes the roll. The undesirable coupled torques affecting the pitch and yaw are then compensated by a feedforward added to a nonlinear state feedback control law [13]. To the best knowledge of the authors this is the first study that includes all the undesirable effects of the thrusters' dynamics and incorporates them in the control scheme with the aim of compensating them. The paper is structured as follows: in section 2 the used prototype is described, in section 3 the equations of the dynamic model and the disturbance effects are presented, the proposed control scheme is explained in section 4 and simulation results are presented and discussed in section 5 . Conclusions are brought in section 6.

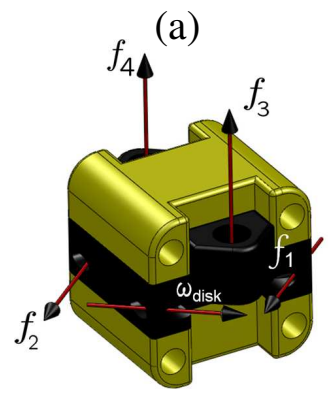

(b)

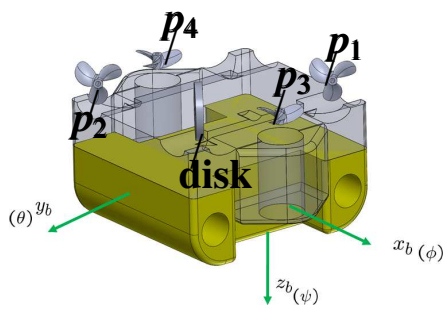

(c)

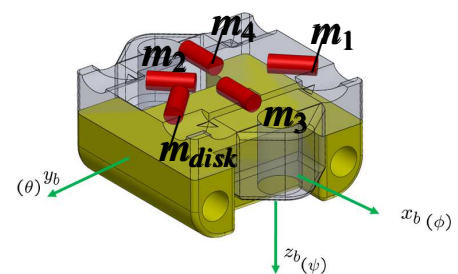

Figure 1: View of the vehicle under study with the orientations of the thrusts and the axis of the disk (a) as well as a transversal cut showing the positions of the propellers, motors, and added disk (b)(c). The body-fixed frame of reference $\left(x_{b} y_{b} z_{b}\right)$ is also shown along with the angle pertaining to each axis $(b)(c)$. 


\section{System Description}

In this paper the vehicle under study is neutrally buoyant, has a cubic shape and weighs $3 \mathrm{~kg}$. This setup correspond to the commercial AC-ROV vehicle (ACCESS Ltd). This latter is equipped with two vertical thrusters (acting on depth and pitch) and four horizontal thrusters (acting on surge, sway, and yaw). As three thrusters are sufficient to control these three latter degrees of freedom, we have replaced one of the four propellers with a disk made of lead as depicted on Fig. 1(b). Gear trains connect each motor to its propeller under an angle of $\pi / 2 \mathrm{rad}$. Fig. 1(a) shows the direction of the thrust exerted by the propellers, as well as the axis of rotation of the added disk. Two transversal cuts of the robot are also shown to present the system components and their configuration. Fig. 1(b) displays the propellers and the disk, whereas Fig. 1(c) displays the orientation of the motors. All the motors are positionned in the $(x, y)$ plane. In this paper, we are interested in the control of the orientation only, the translation will not be treated. The yaw control is provided thanks to the differential speed control of the thrusters 1 and 2. Pitch control is ensured using thrusters 3 and 4, whereas the roll is not actuated by thrusters. The rotational velocity $\omega_{\text {disk }}$ seen in Fig.1(a) refers to the angular velocity of the rotor inserted inside the vehicle. It is coupled to a disk of steel. The dimensions of this disk are to be designed.

$\tau$ is the vector of torques produced by the thrusters to control the orientation angles with $\tau=$ $\left[\begin{array}{lll}0 & \tau_{\text {pitch }} \tau_{\text {yaw }}\end{array}\right]^{T}$. Therefore the control input expressed in N.m is given by:

$$
\tau=T K \omega|\omega|
$$

where $T \in \mathbb{R}^{3 \times 4}$ is the thrusters' configuration matrix taking into account the position and orientation of the propellers. $K$ is the control input coefficient of proportionality between the angular velocity and the obtained torques. $\omega \in \mathbb{R}^{4}$ is the vector of angular velocities of the motors actuating the four propellers, in $\mathrm{rad} . \mathrm{s}^{-1}$. 


\section{Dynamic Modeling of the Underwater Vehicle}

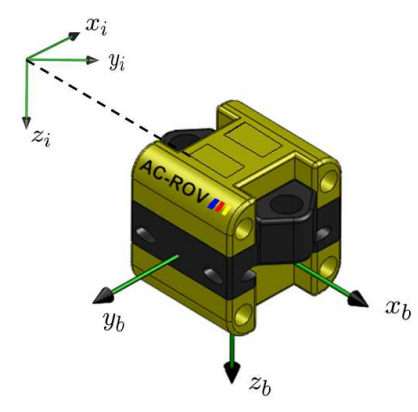

Figure 2: View of the vehicle under study with the body-fixed frame $\left(x_{b} y_{b} z_{b}\right)$ and the earth-fixed frame $\left(x_{i} y_{i} z_{i}\right)$.

\subsection{Background}

The dynamic model used follows the representation shown in Figure 2 and described in [13]. The degrees of freedom under study are: roll, pitch, and yaw. Our dynamic model is written as:

$$
\begin{gathered}
\dot{\eta}=T_{r}(\eta) v \\
M \dot{v}+C(v) v+D(v) v+g(\eta)=\tau+w_{d}
\end{gathered}
$$

where $v=[p, q, r]^{T}, \eta=[\phi, \theta, \psi]^{T}$ are vectors of angular velocities (in the body-fixed frame) and Euler angles (in the earth-fixed frame) respectively. $\operatorname{Tr}(\eta) \in \mathbb{R}^{3 \times 3}$ is the transformation matrix mapping the body-fixed angular velocities to the earth-fixed ones. The model matrices $M(\eta)$, $C(\eta)$, and $D(\eta) \in \mathbb{R}^{3}$ denote inertia (including added mass), Coriolis-centripetal (including added mass), and damping respectively, while $g(\eta)$ is a vector of gravitational/buoyancy forces. $\tau \in$ $\mathbb{R}^{3}$ is the vector of control inputs acting only on pitch and yaw $\left(\tau_{\text {roll }}=0 N\right) . w_{d} \in \mathbb{R}^{3}$ is the vector of disturbances to be detailed here after. In the case of our study, the vehicle used has a 
slow dynamics, and hence it will be moving at velocities low enough to make the Coriolis terms negligible $(C(v) \approx 0)$.

\subsection{Disturbance effects}

In the dynamical model (3), external disturbances coming from the environment are not taken into account and therefore $w_{d}$ only holds the undesirable effects induced by the thursters dynamics. The impact of these effects is put upfront in this paper by considering their models and incorporating them in the vehicle's model. Then, the vector $w_{d}$ takes the following form:

$$
w_{d}=-\tau_{c t_{m}}-\tau_{c t_{p}}-Q-\tau_{G y r o_{m}}-\tau_{G y r o_{p}}
$$

The first two terms represent the inertial counter torques of the motors and propellers respectively. $Q$ is the propeller load torque and the last two terms are the gyroscopic effects produced by the motors and the propellers. Given the configuration of the motors and propellers, we notice that their axes of rotation do not coincide with the ones of the robot. For this reason, all the torques calculated are projected into the robot's frame. This adds coupling and complexity to the system. The details of their computation are given here below:

- Inertial counter torques: This term appears on the rotational axis of each motor and propeller given that they have different axes as illustrated in Fig. 1(b) and Fig. 1(c). It occurs upon a change in the rotational velocity of the motor and propeller generating an opposing resisting torque on the vehicle. It is given by:

$\tau_{c t_{m i}}=J_{m} \dot{\omega}_{i}$ for each motor $i(i=1 \ldots 4)$ and for the disk's motor, with $J_{m}$ the rotor inertia and $\dot{\omega}_{i}$ the time derivative of its angular velocity. Combining the effects of all the motors and projecting them along the robot's axes, we get:

$\tau_{c t_{m}}=\left[\tau_{c t_{\text {mroll }}} \tau_{c t_{\text {mpitch }}} \tau_{c t_{\text {myaw }}}\right]$ with 


$$
\left\{\begin{aligned}
\tau_{c t_{m r o l l}} & =\cos \left(\frac{\pi}{4}\right)\left(\tau_{c t_{m 1}}-\tau_{c t_{m 2}}+\tau_{c t_{m 3}}-\tau_{c t_{m 4}}+\tau_{c t_{m d i s k}}\right) \\
\tau_{c t_{\text {mpitch }}} & =\sin \left(\frac{\pi}{4}\right)\left(-\tau_{c t_{m 1}}+\tau_{c t_{m 2}}+\tau_{c t_{m d i s k}}\right) \\
\tau_{c t_{\text {myaw }}} & =0
\end{aligned}\right.
$$

$\tau_{c t_{\text {myaw }}}$ is null since the motors are in the $(x, y)$ plane and therefore they have no effect on yaw. Similarly, the same equation is applied for each propeller: $\tau_{c t_{p_{i}}}=J_{p} \frac{\dot{\omega}_{i}}{G_{\text {ratio }}}$ with $i(i=1 \ldots 4), J_{p}$ being the propeller inertia and $G_{\text {ratio }}$ the gear ratio between the motor and the propeller. The expression of the vector $\tau_{c t_{p}}$ is not explicitly written since it can be obtained by performing similar projections as for the case of the motors.

- Propeller load torque: The propeller load torque acts on the system in the opposite sense of the propeller angular velocity. It is given as a function of the thrusters' parameters that will be incorporated in our study in a lumped parameter $K_{q}$. We therefore get: $Q_{i}=K_{q}\left|\frac{\omega_{i}}{G_{\text {ratio }}}\right| \frac{\omega_{i}}{G_{\text {ratio }}}$, and $Q=\left[\cos \left(\frac{\pi}{4}\right)\left(Q_{1}-Q_{2}\right), \sin \left(\frac{\pi}{4}\right)\left(Q_{1}-Q_{2}\right),-Q_{3}-Q_{4}\right]^{T}$.

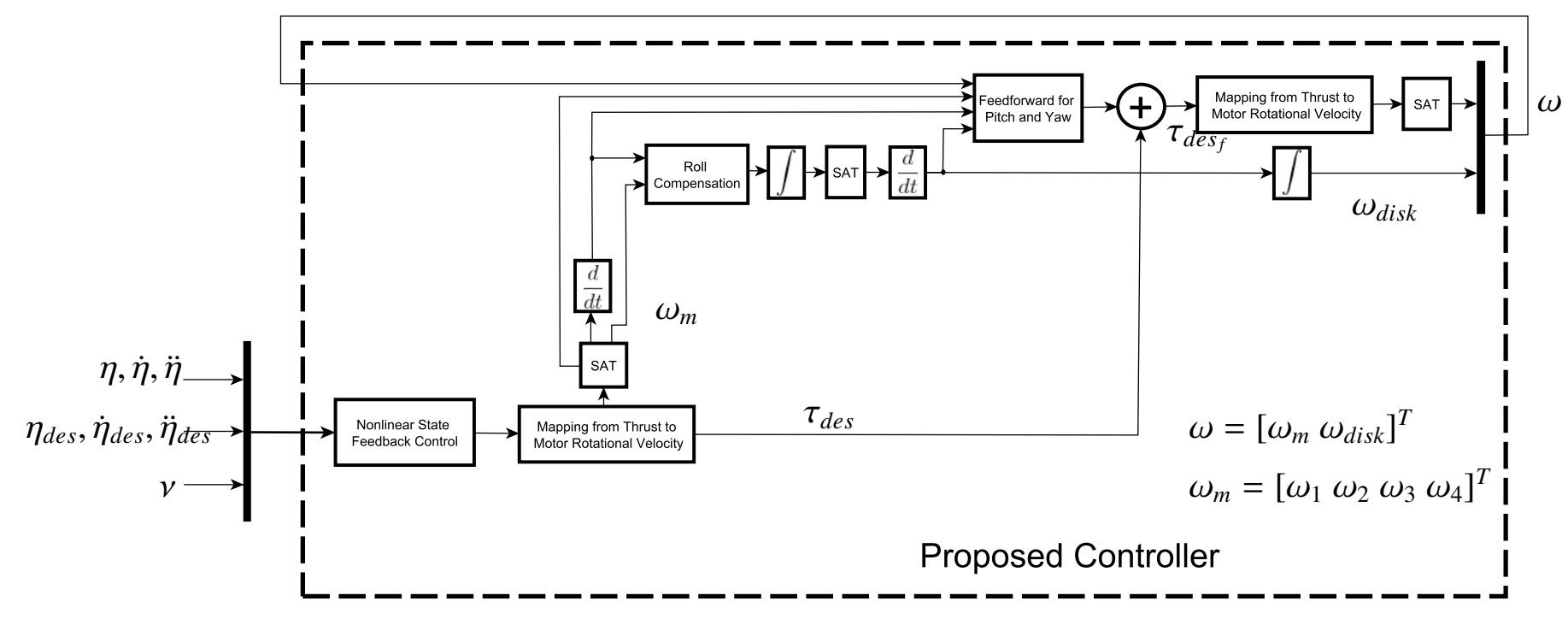

Figure 3: Block diagram of the proposed control scheme.

- Gyroscopic torques: These disturbances are caused by the gyroscopic effect induced when a 
change in the angular momentum of the motors or propellers occurs. It is given by: $\tau_{\text {Gyrom }_{m i}}=$ $J_{m} \omega_{i} \wedge v$ for the motors with $i(i=1 \ldots 4)$ and for the disk's motor, $v=[p, q, r]^{T}$ being the

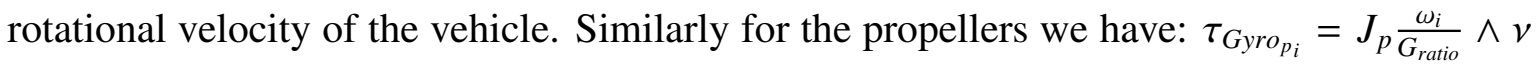
with $i(i=1 \ldots 4)$. In most studies these effects are neglected. However, in this paper, we will demonstrate their impact on the vehicle's dynamics by neglecting them first and then including them.

\section{Proposed Control Scheme}

The model presented in (3) is subjected to various disturbances emanating from its actuation and movements. These undesirable effects have an impact on the performances of the closed-loop system especially when dealing with a small vehicle. Our objective is to design and implement a contol law that will take into account these disturbances and improve the behavior of the vehicle in closed-loop. The proposed control scheme illustrated through the block diagram of Fig. 3 is described in three main parts, namely:

1. Nonlinear State Feedback Control: This controller, as suggested in [13], is applied to the actuated variables of the orientation vector $(\theta$ and $\psi)$.

2. Roll stabilization: In the absence of external disturbances, oscillating effects appear on roll given the coupling between the degrees of freedom due to the configuration of the motors shown in Fig. 1.These oscillations are induced by the propeller load torque and by the inertial counter torque of both motors and propellers. An internal disk is then incorporated to compensate these effects by producing a calculated inertial counter torque.

3. Feedforward Control: This part incorporates the calculated compensation along pitch and yaw of the undesirable torques produced by the thrusters and the compensation of the disturbing effects along the pitch induced by the rotation of the above mentionned disk.

In the following, these three parts of the control scheme will be detailed. 


\subsection{Nonlinear State Feedback Control}

The proposed nonlinear state feedback controller is that suggested in [13] which is based on the linearization of the commanded acceleration $a_{n}$ for a trajectory following in the earth frame. To guarantee that the error converges to zero, $a^{n}$ is then chosen as the following Proportional Integral Derivative (PID) control:

$$
a^{n}=\ddot{\eta}_{d e s}-K_{P} \tilde{\eta}-K_{I} \int_{0}^{t} \tilde{\eta} d t-K_{D} \dot{\tilde{\eta}}
$$

with $\tilde{\eta}=\eta-\eta_{d e s}$ and $\dot{\tilde{\eta}}$ is its first derivative, $\eta_{\text {des }}$ is the desired trajectory and $\ddot{\eta}_{d e s}$ is its second derivative. The computed input is calculated in the body-fixed frame but the trajectory following is performed in the earth-fixed frame and therefore $a^{b}$, the acceleration in the body-fixed frame, is calculated from the simple following transformation:

$$
a^{b}=T_{r}^{-1}(\eta)\left(a^{n}-\dot{T}_{r}(\eta) v\right)
$$

Introducing equation (6) in the dynamic model (3), the control law that cancels the nonlinearities is then chosen to be:

$$
\tau_{d e s}=M a^{b}+C(v) v+D(v) v+g(\eta)
$$

$\tau_{\text {des }}$ being the desired forces and torques to be applied to the vehicle. One remarks that the disturbance term $w_{d}$, explained in (3), is not yet taken into account but will be compensated through the feedforward explained below yielding to the final vector $\tau_{d e s_{f}}$.

\subsection{Disk-based Roll Compensation}

We propose to use the acceleration of the disk's motor as a control input to induce a torque that cancels the effects on the roll. The torque $\tau_{\text {roll }}$ provided by the disk should be equal in magnitude to the disturbing effects and also opposite in direction in order to ensure a complete compensation. From the definitions and equations provided in section 3.B, we get: 
$\tau_{\text {roll }}=-\left(\tau_{c t_{m_{3}}}-\tau_{c t_{m_{4}}}\right)-\cos \left(\frac{\pi}{4}\right)\left(\tau_{c t_{m_{1}}}-\tau_{c t_{m_{2}}}-\tau_{c t_{p_{1}}}+\tau_{c t_{p_{2}}}-Q_{1}+Q_{2}\right)$

Taking into account the configuration of the motor and disk, the inertial counter torque produced by the disk along the roll axis is then expressed by:

$\cos \left(\frac{\pi}{4}\right)\left(-\frac{J_{\text {disk }}}{G_{\text {ratio }}} \dot{\omega}_{\text {disk }}+J_{m} \dot{\omega}_{\text {disk }}\right)=\tau_{\text {roll }}$

We extract from this last equation the acceleration $\dot{\omega}_{d i s k}$ to be applied in order to compensate the effects induced on the roll and stabilize it. However, in many underwater vehicles, (this is the case of the AC-ROV for instance) size constraints do not allow the disk's axis to be parallel to the $x$ axis of the vehicle. This implies a coupled effect on roll and pitch. This problem is overcome thanks to the proposed feedforward described hereafter.

\subsection{Feedforward Compensation of Pitch and Yaw Disturbances}

The vector $\tau_{\text {des }} \in \mathbb{R}^{3}$ is described in (7). The vector of angular velocities $\omega$ can therefore be deduced from (1) and it will be used for the computation of the feedforward control to be summed with $\tau_{d e s}$ in order to compensate the disturbing effects induced by the motors, the propellers, and the disk. Similarly to the compensation of the roll performed above, we compute the torques needed on the pitch and yaw:

- Feedforward for the pitch control input:

$$
\tau_{f f_{\theta}}=-\sin \left(\frac{\pi}{4}\right)\left(-\tau_{c t_{m_{1}}}+\tau_{c t_{m_{2}}}-\tau_{c t_{p_{1}}}+\tau_{c t_{p_{2}}}+\tau_{c t_{d i s k}}-Q_{1}+Q_{2}\right)
$$

- Feedforward for the yaw control input:

$$
\tau_{f f_{\psi}}=-\tau_{c t_{p_{3}}}-\tau_{c t_{p_{4}}}+Q_{3}+Q_{4}
$$

All the terms have been detailed in the previous section. It is worth to note that $\tau_{c t_{\text {disk }}}$ is the counter torque induced by the disk's rotor. We will finally get:

$\tau_{f f}=\left[\begin{array}{ll}0 & \tau_{f f_{\theta}} \tau_{f f_{\psi}}\end{array}\right]^{T}$ and the final expression of the control input becomes $\tau_{d e s_{f}}=\tau_{d e s}+\tau_{f f}$. 

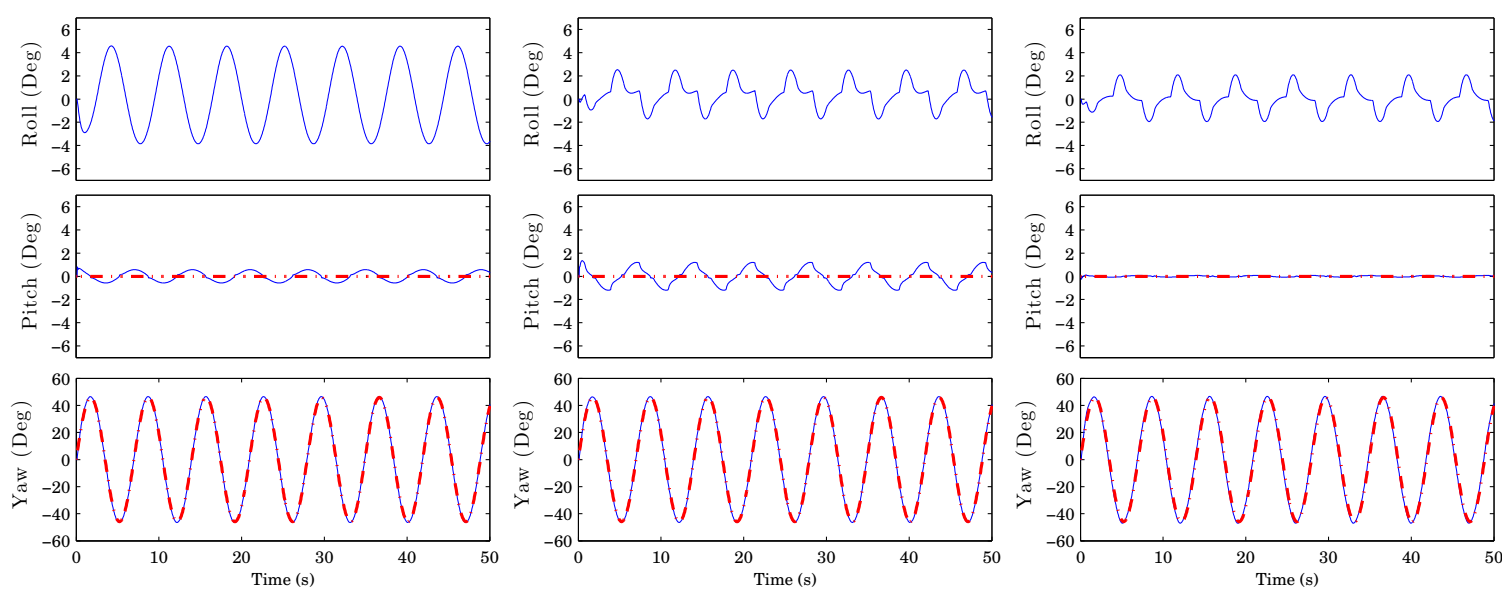

(a) Scenario 1: Nonlinear state(b) Scenario 2: Nonlinear state(c) Scenario 3: Proposed Controller feedback applied on yaw and pitch feedback applied on yaw and pitch with roll stabilization

Figure 4: Time history of the measured orientation angles in blue as well as the desired trajectories in yaw and pitch in dotted red lines for the three scenarios.

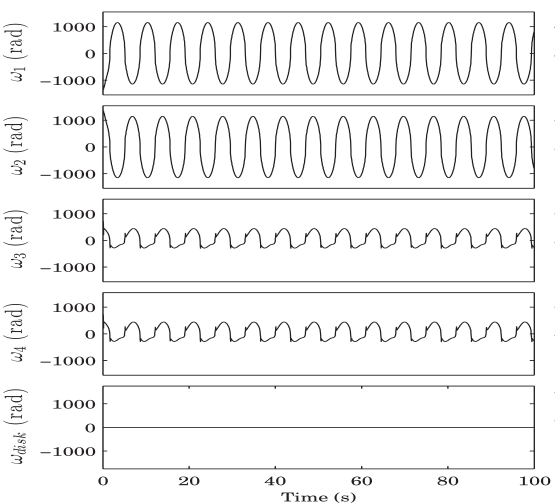

(a)

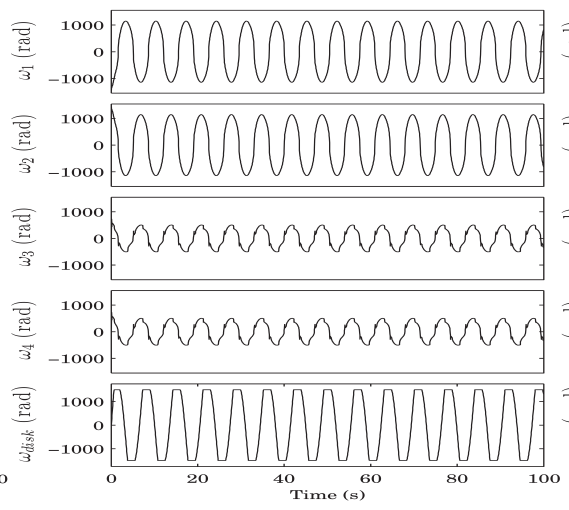

(b)

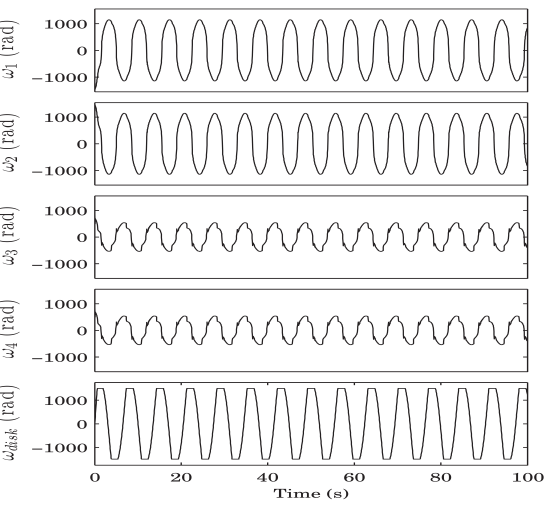

(c)

Figure 5: Angular velocities of the motors in (a) the first scenario, (b) the second scenario and (c) the third scenario.

\section{Numerical Simulations}

The efficiency of the proposed control scheme is put at stake in the following numerical simulations by displaying the time history of the orientation angles. Three scenarios have then been tested, namely: nonlinear state feedback applied on yaw and pitch, nonlinear state feedback applied on yaw and pitch with disk-based roll stabilization, and finally the proposed controller including disk- 
based roll stabilization and feedforward compensation. The input model parameters used in the numerical simulations are summarized in table 1. Simulations have been performed in Matlab software with the well-proven mss simulator [14]. The obtained results are displayed in Fig. 4 along with the control inputs being the torques of the motors (cf. Fig. 5). A desired oscillating trajectory in yaw is generated to put upfront the disturbances on the roll and pitch. These latter two degrees of freedom are stabilized around $0 \mathrm{deg}$. In a fourth scenario, we finally study the influence of the gyroscopic effect and we focus on its dependance with respect to the disk's size.

Table 1: Input model parameters values used in simulations.

\begin{tabular}{clc}
\hline Parameter & Description & Value \\
\hline$J_{m}$ & Rotor inertia & $5.7 \times 10^{-7} \mathrm{~kg} \cdot \mathrm{m}^{2}$ \\
$J_{p}$ & Propeller inertia & $1.59 \times 10^{-6} \mathrm{~kg} \cdot \mathrm{m}^{2}$ \\
$K_{q}$ & Propeller torque coefficient & $9.25 \times 10^{-8} \mathrm{~N} \cdot \mathrm{m} \cdot \mathrm{rad}^{-1} \cdot \mathrm{s}$ \\
$J_{\text {disk }_{\text {small }}}$ & Small disk inertia & $3.52 \times 10^{-5} \mathrm{~kg} \cdot \mathrm{m}^{2}$ \\
$J_{\text {disk }_{\text {medium }}}$ & Medium disk inertia & $4.56 \times 10^{-5} \mathrm{~kg} \cdot \mathrm{m}^{2}$ \\
$J_{\text {disk }_{\text {big }}}$ & Big disk inertia & $5.1 \times 10^{-5} \mathrm{~kg} \cdot \mathrm{m}^{2}$ \\
\hline
\end{tabular}

\subsection{Scenario 1: Nonlinear State Feedback applied on the yaw and pitch}

The aim of this scenario is to show the induced effects of the motors and propellers on the roll and pitch when the roll is not controlled. The pitch is controlled to remain stable around 0 deg while a desired trajectory oscillating from $-45 \mathrm{deg}$ to $+45 \mathrm{deg}$ is imposed on the yaw. This persistent oscillation is intentionally made to observe the induced disturbances on the remaining degrees of freedom in orientation. Fig. 3(a) displays the evolution of the three orientation angles under study. In this example, we observe that the yaw follows the desired trajectory in closed-loop under the nonlinear state feedback controller, while the pitch despite being controlled, exhibits some minor residual oscillations ranging from $-0.6 \mathrm{deg}$ to $+0.6 \mathrm{deg}$. Due to the configuration of the thrusters, the roll is not actuated. However, thanks to the positive metacentric distance, the roll angle remains 
naturally stable with oscillations varying from -4 to $+4.5 \mathrm{deg}$. Fig. 4(a) shows the torques of the motors. Motors 1 and 2 controlling the yaw have an angular velocity of around $1100 \mathrm{rad} / \mathrm{s}$ whereas motors 3 and 4 stabilizing the pitch have angular velocities of around $300 \mathrm{rad} / \mathrm{s}$. In this scenario the disk's motor is kept off.

\subsection{Scenario 2: Nonlinear State Feedback applied on yaw and pitch with disk-based roll stabilization}

In this scenario, the objective is to control all the degrees of freedom pertaining to the orientation. The desired trajectory in yaw is the same as the previous scenario, while the pitch is stabilized around $0 \mathrm{deg}$. These two degrees of freedom are controlled using the nonlinear state feedback controller. The roll is stabilized thanks to the effect of the disk's acceleration. This latter cancels out the disturbances caused by the coupled effects of the thursters. Fig. 3(b) displays the evolution of the orientation angles. In this example, we can clearly see that the amplitude of the oscillations in pitch is increased in the closed loop response (from $-1.2 \mathrm{deg}$ to $+1.3 \mathrm{deg}$ ) in comparison with the previous scenario. This is due to the effects induced by the disk and its associated motor. However, one observes that the effects on the roll were reduced by half compared to the previous scenario, that is, current oscillations are ranging from $-1.7 \mathrm{deg}$ to $+2.5 \mathrm{deg}$. The rotational velocities of the motors 1 and 2 in this scenario are similar to the previous case (cf. Fig. 4(b)), motors 3 and 4 controlling the pitch have an increased rotational velocity of $500 \mathrm{rad} / \mathrm{s}$. The motor's disk saturates at the maximum allowed rotational velocity, being $1500 \mathrm{rad} / \mathrm{s}$.

\subsection{Scenario 3: Proposed Control Scheme Including Disk-based Roll Stabilization and Feedforward Compensation}

The results of the proposed control scheme, detailed in section 4, are displayed in Fig. 3(c). The difference with respect to the previous scenario is that a feedforward has been added on the pitch and yaw. The roll angle exhibits a similar behavior compared to the previous case and the yaw 
angle still follows the desired trajectory. However, the pitch angle is stabilized close to 0 deg. The thrusters' effects are therefore completely compensated on this latter degree of freedom with the addition of the feedforward. The motors have a very similar consumption compared to the previous scenario (cf. Fig. 4(c)). The rotational velocities of motors 3 and 4 increase to $600 \mathrm{rad} / \mathrm{s}$.

\subsection{Scenario 4: Disk Size and Gyroscopic Effect}

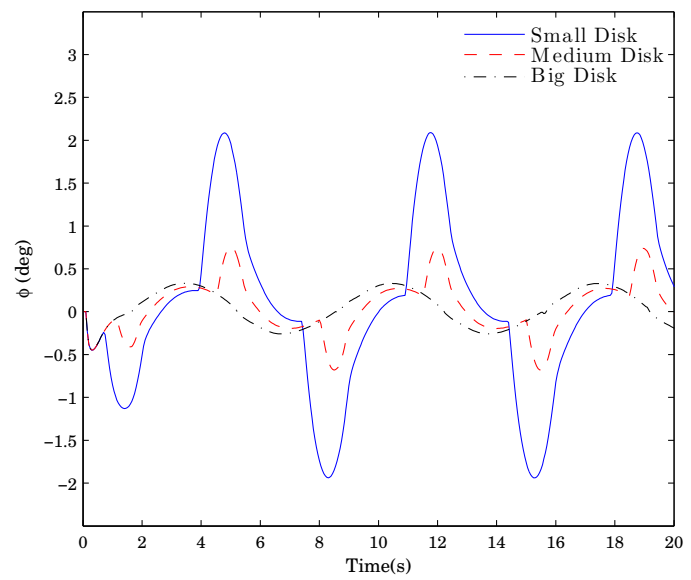

Figure 6: Time history of the roll angle depending on the disk's size (neglecting gyroscopic effect)

It has to be noticed that with a bigger disk a better stabilization of roll can be expected. Fig. 6 shows the evolution of the roll angle for three different disk sizes. It was stated in section 3 that the gyroscopic effects are negligible, which was applicable in the absence of the internal disk or in the presence of a small one. However, with a bigger sized disk turning at a more important angular velocity, the gyroscopic effect cannot be neglected anymore. Fig. 7 displays the roll angle when the proposed control law was applied in the case of a small disk (cf. Fig. 7(a) ) and a big disk (cf. Fig. 7(b)). The red dotted lines in Fig. 7 refer to the case with gyroscopic effect in the model, and the blue solid lines refer to the case when these effects are neglected. When the gyroscopic effect is taken into account, we observe that the peak to peak amplitude of the roll angle remains unchanged in the case of a small-sized disk (peak to peak amplitude of 4 degrees), even though the oscillations are shifted. However, in the case of a big-sized disk, we notice that the peak to peak 


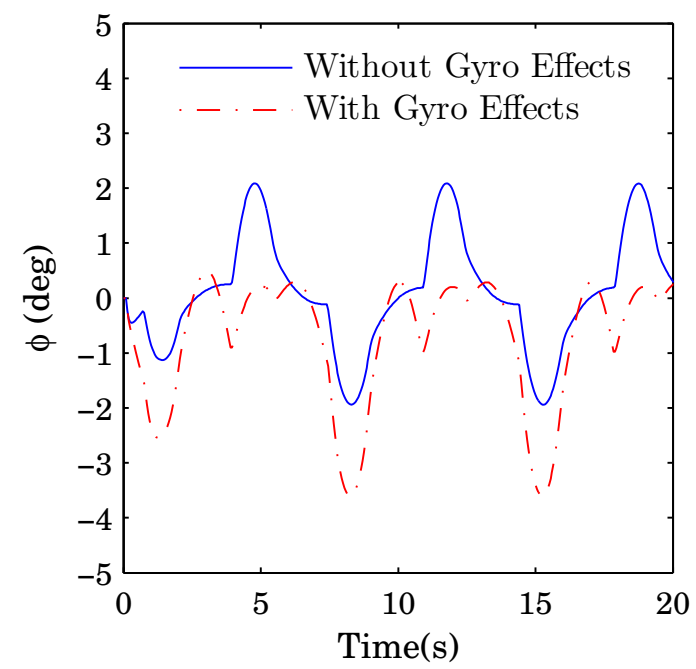

(a)

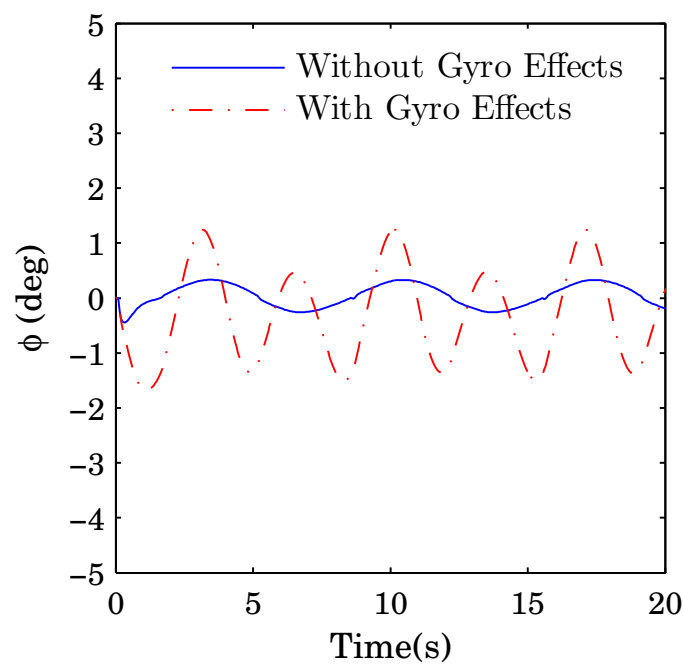

(b)

Figure 7: Roll angle in presence of a small disk (a) and a big disk (b) with overlapping plots both neglecting or not the gyroscopic effects.

amplitude of the roll angle becomes five times larger than when neglectig gyroscopic effect (the oscillations varying from $-0.3 \mathrm{deg}$ to $+0.3 \mathrm{deg}$ increase to $-1.8 \mathrm{deg}$ to $+1.2 \mathrm{deg}$ ). This observation is important and should be considered when choosing the disk. Indeed, there is no optimal choice for the dimension and weight of the disk. The choice depends on both technological constraints (allowed power, size, and load) and application related requirements. As can be seen on Fig. 7, although a bigger disk decreases the error bounds, the gyroscopic effect involves high frequency oscillations, which could be a problem for example for imaging or manipulation purposes. The best way to proceed is to set the desired bounds for the roll error and to choose the smallest sized disk accordingly. For this purpose, the maximal dynamical effects induced by thrusters (see section 3) have to be computed according to the motors' features. Then, knowing the maximal acceleration allowed by the disk's motor, one can easily compute the minimal value of the disk's inertia. Choosing this value will limit the undesirable oscillations induced by the gyroscopic effect. 


\section{Conclusion}

This paper introduces a novel concept for roll stabilization of an underactuated underwater vehicle. A new control architecture for the orientation of an underactuated underwater vehicle is presented. It is based on a nonlinear state feedback controller augmented by a feedforward control for the pitch and yaw. The unactuated roll is stabilized using the inertial counter torques induced by an internal disk actuated by a motor. A complete study of the disturbances emanating from the motors and affecting the robot's orientation was presented. Numerical simulations have shown the effectiveness of the proposed scheme through the obtained promising results. Future work will be focused on implementing this approach on the prototype of an underwater vehicle. We will also study the influence of the disk's size on the traking performance in pitch.

\section{References}

[1] P Mulhaupt, B. Srinivasan, J Levin, and D Bonvin. Cascade control of the toycopter. In European Control Conference, Kalsruhe, Germany, 1999.

[2] Naomi Ehrich Leonard. Stabilization of steady motions of an underwater vehicle. In Proceedings of the 35th IEEE Conference on Decision and Control, pages 3980-3985, 1996.

[3] Naomi Ehrich Leonard. Stability of a bottom-heavy underwater vehicle. Automatica, 33(3):331-346, 1997.

[4] Naomi Ehrich Leonard. Stabilization of underwater vehicle dynamics with symmetrybreaking. Systems and Control Letters, 32:35-42, 1997.

[5] Naomi Ehrich Leonard and Jerrold E. Marsden. Stability and drift of underwater vehicle dynamics: Mechanical systems with rigid motion symmetry. Physica D, 105:130-162, 1997. 
[6] Naomi Ehrich Leonad and Cragi A. Woolsey. Internal actuation for intelligent underwater vehicle control. In Tenth Yale Workship on Adaptive and Learning Systems, 1998.

[7] Craig A. Woolsey and Naomi Ehrich Leonard. Underwater vehicle stabilization by internal rotors. In Proceedings of the 1999 American Control Conference, pages 3417-3421, 1999.

[8] Craig A. Woolsey and Naomi Ehrich Leonard. Global asymptotic stabilization of an underwater vehicle using internal rotors. In Proceedings of the 38th Conference on Decision and Control, pages 3417-3421, Phoenix, Arizona USA, 1999.

[9] Craig A. Woolsey and Naomi Ehrich Leonard. Stabilizing underwater vehicle motion using internal rotors. Automatica, 38(12):2053-2062, 2002.

[10] C. Shlutz and C.A Woolsey. An experimental platform for validating internal actuator control strategies. In Proceedings of GCUV, pages 209-214, 2003.

[11] Blair Thornton, Tamaki Ura, Yoshiake Nose, and Stephen Turnock. Internal actuation of underwater robots using control moment gyros. In Oceans, pages 591-598, 2005.

[12] Blair Thornton, Tamaki Ura, Yoshiake Nose, and Stephen Turnock. Zero-g class underwater robots and unrestricted attitude control using control moment gyro. In Oceans, pages 1-5, 2006.

[13] T.I. Fossen. Marine Control Systems:Guidance, Navigation and Control of Ships, Rigs and Underwater Vehicles. Marine Cybernetics, As, Trondheim, 2002.

[14] Thor I. Fossen and Tristan Perez. Marine Systems Simulator (mss). http://www.marinecontrol.org/, 1991.

\section{Biographies}


Divine MAALOUF received her $\mathrm{PhD}$ degree in 2013 in underwater robotics from the University of Montpellier 2, France. She completed her first year of masters in the University of Genova, Italy in 2009 and the second one in Ecole Centrale de Nantes, France in 2010 as part of the Erasmus Mundus Grant for the master Program EMARO (European Masters for Advanced Robotics). She is currently working as a control engineer at Kietta SAS. Her research interests include dynamic modeling, nonlinear control and underwater robotics.

Vincent CREUZE received his PhD degree in 2002 in robotics from the University Montpellier 2, France. He is currently an associate professor at the University Montpellier 2, attached to the Robotics Department of the LIRMM (Montpellier Laboratory of Computer Science, Robotics, and Microelectronics). His research interests include design, modelling, and control of underwater robots, as well as underwater computer vision.

Ahmed CHEMORI received his MSc and PhD degrees respectively in 2001 and 2005, both in automatic control from the Grenoble Institute of Technology. He has been a Post-doctoral fellow with the Automatic control laboratory of Grenoble in 2006. He is currently a tenured research scientist in Automation and Robotics at the Montpellier Laboratory of Computer Science, Robotics, and Microelectronics. His research interests include nonlinear, adaptive and predictive control and their applications in humanoid robotics, underactuated systems, parallel robots, and underwater vehicles.

Olivier TEMPIER received his Master of Engineering in electrical engineering and industrial data processing in 1999. From 1999 to 2006, he worked in the industry as a R\&D engineer (demining robot, radar system, maritime security...) Since 2006, he works in the Robotics Department of the LIRMM (Montpellier Laboratory of Computer Science, Robotics, and Microelectronics). 